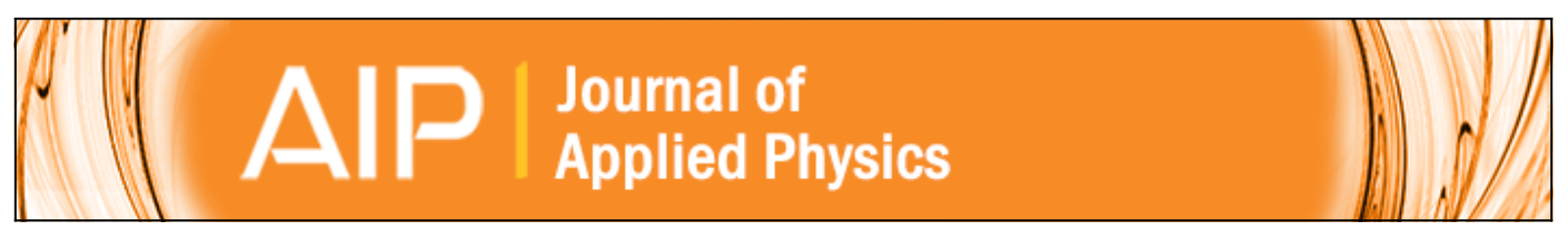

\title{
Design of a transverse flux machine for power generation from seawaves
}

Vicenzo Franzitta, Alessia Viola, and Marco Trapanese

Citation: Journal of Applied Physics 115, 17E712 (2014); doi: 10.1063/1.4865883

View online: http://dx.doi.org/10.1063/1.4865883

View Table of Contents: http://scitation.aip.org/content/aip/journal/jap/115/17?ver=pdfcov

Published by the AIP Publishing

\section{Articles you may be interested in}

Study of the foundation design for a linear generator wave energy converter using stochastic methods

J. Renewable Sustainable Energy 7, 063112 (2015); 10.1063/1.4936420

Constrained optimal control of a point absorber wave energy converter with linear generator

J. Renewable Sustainable Energy 7, 043127 (2015); 10.1063/1.4928677

Algorithm for the calculation of the translator position in permanent magnet linear generators

J. Renewable Sustainable Energy 6, 063102 (2014); 10.1063/1.4900553

Evaluation of wave energy generation from buoy heave response based on linear generator concepts

J. Renewable Sustainable Energy 4, 063137 (2012); 10.1063/1.4771693

A resonant two body system for a point absorbing wave energy converter with direct-driven linear generator J. Appl. Phys. 110, 124904 (2011); 10.1063/1.3664855

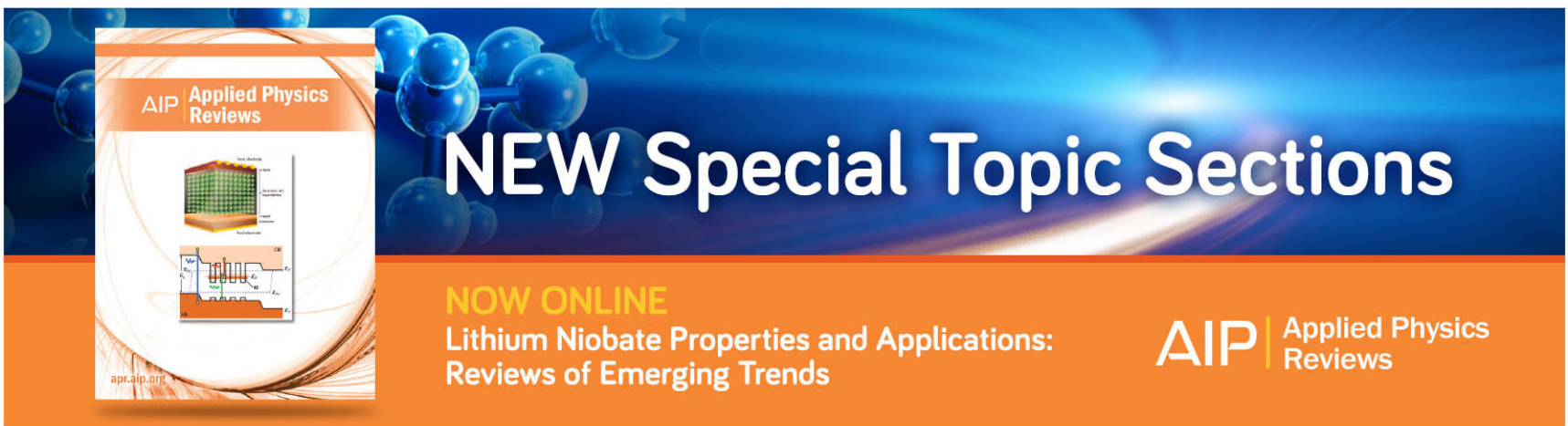




\title{
Design of a transverse flux machine for power generation from seawaves
}

\author{
Vicenzo Franzitta, Alessia Viola, ${ }^{\text {a) }}$ and Marco Trapanese \\ DEIM, Palermo University, Palermo I-90128, Italy
}

(Presented 6 November 2013; received 23 September 2013; accepted 12 November 2013; published online 21 February 2014)

\begin{abstract}
In this paper, we present a transverse flux linear generator. We investigate the possibility to use this generator to extract energy from seawaves. We propose an optimization procedure that allows us to obtain an optimized design of the generator. The optimized design of the converter shows a power generation capability index much higher than other renewable systems. (C) 2014 AIP Publishing LLC. [http://dx.doi.org/10.1063/1.4865883]
\end{abstract}

\section{I.INTRODUCTION}

Linear electrical generator has been recently studied for the exploitation of sea wave energy, however, the definition of the optimum geometry to be used is still debated and due to the fact that seawave energy is characterized by low speed and very high force, a generator which is able to convert energy at low speed and high force is needed. In this paper, we investigate the possibility to use a transverse flux linear generator (TFG) because transverse flux technology presents the highest force density per volume index among the iron based electrical machines. The advantages of TFG topology against the classical longitudinal concept are: ${ }^{1}$ (a) the magnetomotive force per pole is independent from the total pole numbers; (b) the magnetic flux geometry and the coil section are independent design parameters; (c) armature coils geometry is simple; and (d) phases are magnetically decoupled. In order to exploit these advantages, we have designed a TFG for power generation from seawaves.

The generator has been preliminarily designed following the approach presented in Ref. 2. The optimization has been performed by letting some geometrical parameters be varied in order to achieve the maximum force and the maximum energy output per unit of material used in the assembly of the generator. However, since the magnetic flux path is intrinsically 3D, a 3D finite element analyses has been used to verify the design of the motor. In order to optimize the design, several simulations were done assuming several types of seawaves hitting the generator. In each simulation, the electrical and geometrical parameters of the generator were varied and rotor was supposed to be acted by a water wave train. The wave train consisted of ten oscillations. Each oscillation of the train wave had an amplitude and a frequency generated through a random number generator, whose statistic features were obtained from the experimental data of actual seawaves. This approach leads to determine a well defined geometry of TFG. This geometry has been used to numerically simulate the generated electromotive force, the power output, and to evaluate the maximum mechanical stress on the generator's parts.

\footnotetext{
${ }^{a)}$ Author to whom correspondence should be addressed. Electronic mail: marco.trapanese@unipa.it. Present address: DEIM, viale delle Scienze ed. 9, Palermo University, Palermo I-90128, Italy. Fax: +39-091488452.
}

This paper is organized as follows: in Sec. II, the characteristics of the primary source of energy (seawave) and the proposed method to capture this energy is briefly presented; in Sec. III, a design of the machine and some numerical evaluation are reported; in Sec. IV, a detailed analysis of the numerical simulation along with experimental results is presented, and finally in Sec. V, the conclusions are drawn.

\section{PRINCIPLE OF OPERATION FOR THE GENERATION OF ELECTRICAL ENERGY FROM SEAWAVES}

The principle of operation of the generator is shown in Fig. 1.

The moving part of the generator is driven from the sea waves and induces and emf on the winding mounted on the armature.

The motion of the slider shown in Fig. 1 can be modelled as follows:

$$
\begin{gathered}
\rho g S\left(y_{s}-y_{g}\right)-m g-k i b-k_{s}\left(y_{s}-y_{0}\right)=m \frac{\partial^{2} y_{g}}{\partial t^{2}}, \\
e(t)=i R_{i}+L \frac{\partial i}{\partial t}+i R, \\
e(t)=-\frac{\partial \varphi(t)}{\partial t},
\end{gathered}
$$




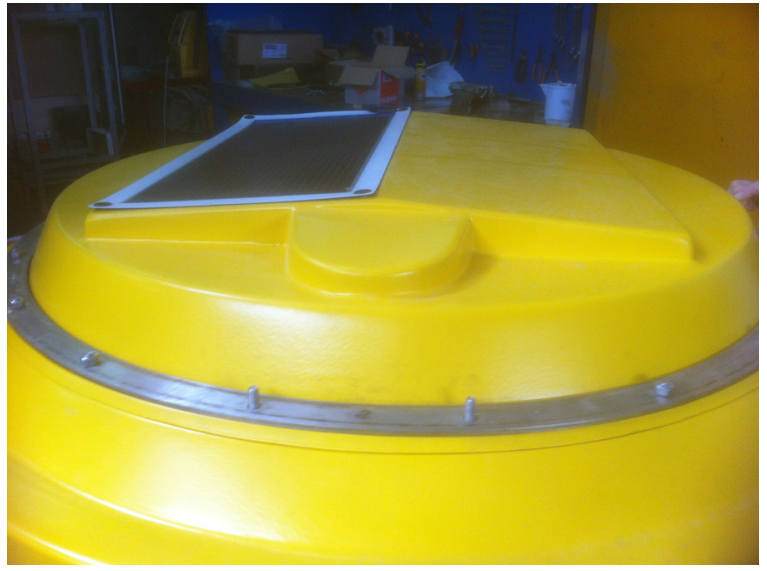

FIG. 2. The measurement buoyant.

where $\mathrm{g}$ is the acceleration gravity, $\rho$ is the water density, $\mathrm{S}$ is the area of the buoyant, $y_{s}$ is the vertical coordinate describing the sea level, $\mathrm{y}_{\mathrm{g}}$ is the vertical coordinate describing the position of the slider, $\mathrm{k}_{\mathrm{s}}$ is the spring constant, $\mathrm{m}$ is the total mass of the translator and of the buoyant, $\mathrm{k}$ is the electromagnetic constant, $\mathrm{i}$ the current $\mathrm{B}$ the magnetic induction, $e(t)$ is the induced electromotive force (emf), $R_{i}$ is the internal resistance of the machine, $\mathrm{L}$ is the inductance of the machine, $R$ is the resistance of the external load, $f$ is the linked flux, and $y_{0}$ is the rest position. The definition of the coordinates can be seen in Fig. 1.

The first equation models the dynamic behaviour of the device. On the left hand side there are the forces acting on the slider and on the right hand side there is the acceleration of the slider. No hydraulic friction is considered.

The second equation describes in terms of lumped parameters the generator connected to a resistive load.

The third equation describes the emf.

In order to optimize the generator for the conversion of energy contained in seawaves, a detailed knowledge of the characteristics of the seawaves that will interact with the generator is needed. These characteristics strongly depend on geographical site as a result in order to acquire this knowledge a measurement system has been built and placed in the area of installation of the generator. In Fig. 2 , the measurement system is shown. The characteristics of the seawaves are shown in Fig. 3, where a typical waveform is shown. From Fig. 3, it can be deducted that the main problem in order to perform the optimization is to model realistically the motion of the sea. However, the main features of seawave (maximum vertical speed and wave height and wave period) have been used for the preliminary design.

In order to have a usable numerical model, it has been assumed that the waves hitting the generator consisted of purely sinusoidal waves but each waves had different amplitude and period in comparison with the others. The amplitudes and the periods were statistically distributed accordingly with the experimental data reported in Ref. 3.

As a result, in the proposed approach, each seawave has been modelled as follows:

$$
y_{s}=A \sin (\omega t)+y_{0},
$$

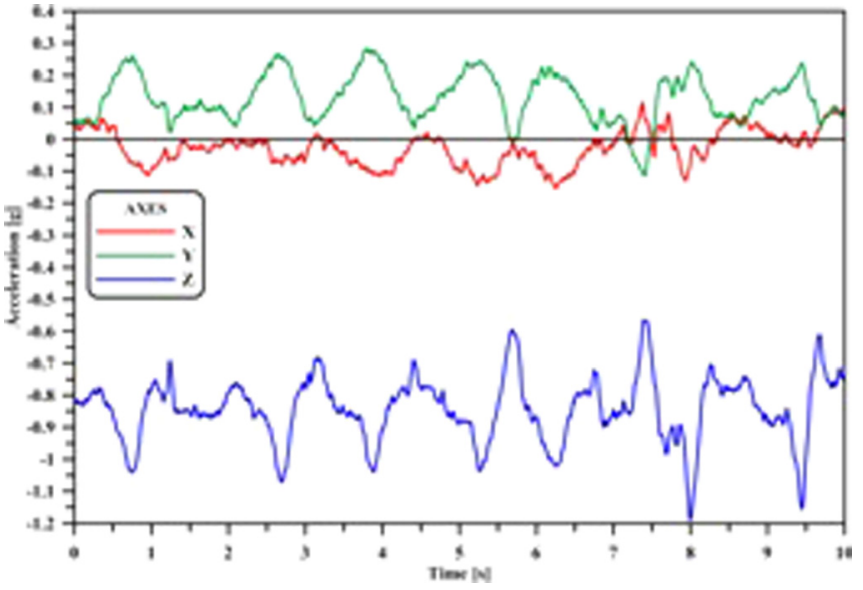

FIG. 3. A typical seawave measurement.

where $\mathrm{A}$ is the amplitude and $\omega$ is the frequency. The use of such a driving force does not allow to use an analytical approach to the optimization, but a numerical approach which includes the main stochastic feature of the phenomenon must be followed.

\section{DESIGN OF A TRANSVERSE FLUX GENERATOR}

The seawave characteristics presented in Sec. II clearly show that the energy in seawaves is mostly "contained" in terms of high force density and low speed. This form of energy is not very usual for electromagnetic devices that are more aimed to convert energy from relatively high speed and less intense force. As a result, the direct conversion of seawaves energy into electrical energy is a difficult task, which needs electromagnetic converters specifically conceived for this purpose. The main characteristic that such a converter should have is the capability to handle high force density. From this point of view, the most suitable electric machine able to deal with high density force is a transverse flux linear machine and as a result in this paper, we propose to use a Double Sided Transverse Flux Linear Synchronous Generator as the electromagnetic converter to be used to convert seawave energy into electric energy. It is shown in Fig. 4. This kind of machine has been proposed recently as motor. ${ }^{2,4}$ Fig. 5 shows the structure of the three phase unit and its parameter specifications. The machine consists of a moving armature and a standing excitation. The armature is composed of four coils and an iron core. The two coils on one side of the armature unit are wound in the opposite direction of the coils wound on the other side and are
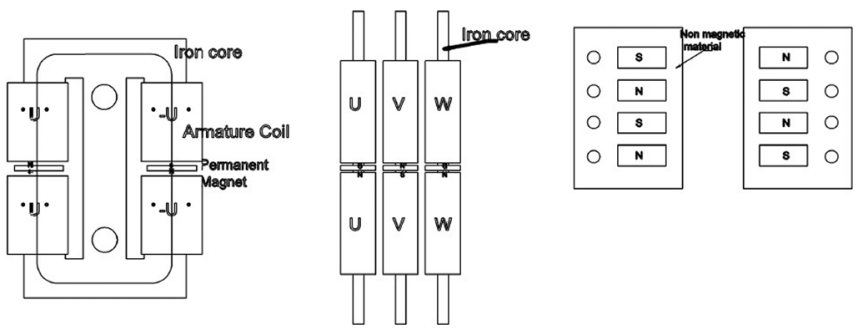

FIG. 4. The basic configurations of the three phase unit. (a) Armature and field inductor. (b) Configuration along the moving direction. 

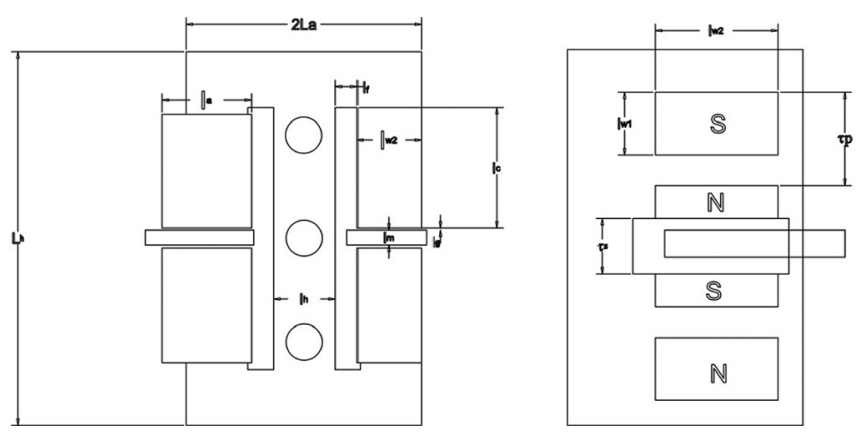

FIG. 5. Specifications of geometrical parameters.

connected in series. The coils of different armature units are connected in parallel. Armature units and inductor magnets are installed along the moving direction. Magnets are made of $\mathrm{NdFeB}$ and are separated by 180 electrical degrees. Each armature unit is separated by the following one by 120 electrical degree. The main difference between this transverse machine and other transverse conventional solutions is that in this case the magnetic circuit is inherently $2 \mathrm{D}$.

The 2-dimensionality of the magnetic circuit simplifies the following aspects of the manufacturing aspects of the TFG:

1) the iron core can be assembled from traditional laminated steel plates;

2) the structure is simple and can be fabricated in sub unit;

3) higher manufacturing tolerances which allows a simplification in the air gap design and fabrication.

In Ref. 2, it is shown how the proposed generator experiences a maximum force expressed as follows:

$$
F_{t_{\max }}=p \frac{E_{r m s}}{v} I=\frac{\sqrt{2}\left(p \pi \varphi_{m l_{\_} \max }\right)}{2 t_{p}} N\left(l_{a}, l_{c}\right) I
$$

where the geometric parameters $l_{a}$ and $l_{c}$ can be deducted from Figs. 4 and 5.

\section{OPTIMIZATION PROCEDURE AND NUMERICAL SIMULATION}

Optimization procedures have been focused on the maximization of the annual production yield per unit of active (iron and magnets) material that has been used. This approach is equivalent to design the generator maximizing the tangential force and leads to an expression for force as in (4). However, expression in (4) can been more exactly computed if a 3D analysis is performed and if some numerical simulation of the generator lead by seawaves can be performed. In each simulation, parameters $\mathrm{R}_{\mathrm{i}}, \mathrm{L}, \mathrm{k}$, and $\phi$ had values different from the ones used in the previous simulation. In each run of the simulation the water was let vary according to Eq. (2) and a wave train was generated. The wave train consisted of ten oscillations. Each oscillation of the train wave had an amplitude and a frequency generated through a random number generator, whose statistic features were obtained from the experimental data above mentioned. $\mathrm{R}_{\mathrm{i}}, \mathrm{L}, \mathrm{k}$, and $\phi$ were let vary among some values that were
TABLE I. Preliminary design specifications.

\begin{tabular}{lccccc}
\hline \hline Symbol (mm) & Quantity & Symbol (mm) & Quantity & Symbol & Quantity \\
\hline $\mathrm{L}_{\mathrm{a}}$ & 40 & $\mathrm{l}_{\mathrm{f}}$ & 10 & $1_{\mathrm{g}}[\mathrm{mm}]$ & 1 \\
$\mathrm{~L}_{\mathrm{b}}$ & 100 & $\mathrm{l}_{\mathrm{b}}$ & 20 & $\mathrm{l}_{\mathrm{b}}[\mathrm{mm}]$ & 2 \\
$\mathrm{p}$ & 13.5 & $\mathrm{~L}_{\mathrm{w} 1}$ & 11.5 & $\mathrm{l}_{\mathrm{a}}, \mathrm{l}_{\mathrm{m}}, \mathrm{l}_{\mathrm{c}}$ & Variables \\
$\mathrm{s}$ & 9 & $\mathrm{~L}_{\mathrm{w} 2}$ & 20 & & \\
\hline \hline
\end{tabular}

compatible with the size of the linear generator, with the induced emf and with the linked flux.

The design specifications are reported in Table I from where it can be seen that the free parameters were $l_{\mathrm{a}}$ and $l_{\mathrm{c}}$.

The objective function was the energy output that under the assumptions presented has the following form:

$$
E_{o}=\int_{0}^{T_{f i n}} i^{2} \cdot R d t
$$

where $E_{o}$ is the output energy and $T_{\text {fin }}$ is the final time. The final $\mathrm{T}$ could vary from run to run and it was equal to the time needed to let the water complete 10 full oscillations. The output of the optimization procedure was the values of the design parameters $l_{a}$ and $l_{c}$.

This procedure led to compute the free parameters $l_{a}$ and $l_{c}$, which were equal to $2.5 \mathrm{~mm}$ and $4 \mathrm{~mm}$.

In order to verify if the design procedure had led to satisfactorily results, we computed thrust at the optimal point by using Eq. (3) and computed the same quantity obtained by a $3 \mathrm{D}$ simulation. The theoretical value that was computed was $38.3 \mathrm{~N}$, the value obtained in the 3D FEM analysis was $36.8 \mathrm{~N}$.

From this value, we were able to compute the power density generation capability of the device that was equal (for the type of seawaves considered in the simulation) to $6.5 \mathrm{~kW} / \mathrm{m}^{2}$. This value is much higher of a typical Photovoltaic system.

\section{CONCLUSIONS}

In this paper, we investigated the possibility to use a TFG to convert seawave energy into electrical energy. We proposed a possible design and an optimization procedure have been illustrated. The optimized design of the converter shows a power generation capability index much higher than other renewable systems.

\section{ACKNOWLEDGMENTS}

This work was funded by IMPETUS project.

${ }^{1}$ J. S. D. Garcia, M. V. F. da Luz, J. P. A. Bastos, and N. Sadowski, "Transverse flux machines: What for?," IEEE Multidiscip. Eng. Educ. Mag. 2(1), 4-6 (2007).

${ }^{2}$ J. S. Shin, T. Koseki, and H. J. Kim, "Proposal of double sided transverse flux linear synchronous motor and a simplified design for maximum thrust in nonsaturation region,” IEEE Trans. Magn. 49(7), 4104-4108 (2013).

${ }^{3} \mathrm{M}$. Trapanese, "Optimization of a sea wave energy harvesting electromagnetic device," IEEE Trans. Magn. 44(11), 4365-4368 (2008).

${ }^{4}$ V. Di Dio, V. Franzitta, F. Muzio, G. Scaccianoce, and M. Trapanese, "The use of sea waves for generation of electrical energy and hydrogen," in MTS/IEEE Biloxi - Marine Technology for Our Future: Global and Local Challenges, OCEANS, 2009. 